\title{
DESCRIPCIÓN DE LOS ESTADOS INMADUROS DE LIGYRUS NASUTUS (BURMEISTER, 1847) (COLEOPTERA: MELOLONTHIDAE: DYNASTINAE) CON INFORMACIÓN SOBRE SU BIOLOGÍA
}

\author{
Concepción Ramírez-Salinas', Cutberto PaCheco-Flores², \\ Adriana E. CASTRO-RAMírez' ${ }^{1}$ Miguel Ángel MORóN3 ${ }^{3}$
}

1El Colegio de La Frontera Sur (ECOSUR). Carretera Panamericana y Periférico Sur s/n.

San Cristóbal de Las Casas, Chiapas, 29290 MÉXICO.

2Universidad Intercultural de1 Estado de Guerrero, La Ciénega, Malinaltepec, Guerrero, MÉXICO.

3Instituto de Ecología, A.C. A. P. 63 Xalapa, Veracruz. E-mail: miguel.moron@inecol.edu.mx

Ramírez-Salinas C., C. Pacheco-Flores, A. E. Castro-Ramírez \& M. Á. Morón. 2010. Descripción de los estados inmaduros de Ligyrus nasutus (Burmeister, 1847) (Coleoptera: Melolonthidae: Dynastinae) con información sobre su biología. Acta Zool. Mex. (n. s.), 26(3): 617-625.

RESUMEN. Se describe la larva de tercer estadio de Ligyrus nasutus (Burmeister) con base en cinco ejemplares procedentes de parcelas agrícolas en Villa Flores, Chiapas, México. También se describe la pupa de L. nasutus con base en ejemplares obtenidos durante el desarrollo de las larvas en cautiverio. Se incluyen ilustraciones de los caracteres diagnósticos, datos sobre su biología y una clave para separar las larvas conocidas del género.

Palabras clave: "gallina ciega", larvas saprófagas, fauna de suelo, Chiapas, México.

Ramírez-Salinas C., C. Pacheco-Flores, A. E. Castro-Ramírez \& M. Á. Morón. 2010. Description of immature stages of Ligyrus nasutus (Burmeister, 1847) (Coleoptera: Melolonthidae: Dynastinae) with information on its biology. Acta Zool. Mex. (n. s.), 26(3): 617-625.

ABSTRACT. The third instar larva of Ligyrus nasutus Burmeister is described based on five specimens collected from agricultural fields, where cattle fed with stubble from the previous cycle, and pastures with cattle in Villa Flores, Chiapas, Mexico. The pupa of L. nasutus is also described using specimens obtained from rearing in captivity. Drawings of the diagnostic structures, biological data and a key to the known larvae of the species of Ligyrus are included.

Keywords: white grubs, saprophagous larvae, soil fauna, Chiapas, Mexico.

\section{INTRODUCCIÓN}

Ligyrus nasutus habita en bosques tropicales caducifolios y comunidades secundarias situadas entre el nivel del mar y los $1000 \mathrm{~m}$ de altitud en México, Guatemala, El Salvador, Honduras, Nicaragua y Costa Rica (Morón et al.,1997). En México se ha registrado en los estados de Campeche, Guerrero, Jalisco, Michoacán, Oaxaca, Quintana Roo, Tamaulipas y Yucatán. En Chiapas se ha encontrado en

Recibido: 26/01/2010; aceptado: 11/06/2010. 
Tuxtla Gutiérrez, Ocozocuautla, Motozintla, Chiapa de Corzo, Comitán, Laguna Bélgica, El Aguacero y Villa Las Rosas (Morón et al., 1997; Alcázar-Ruiz et al., 2003; Morales-Morales et al., 2005). En una muestra de 6,780 escarabajos adultos atraídos por las trampas de luz y el alumbrado público en el municipio de Villa Flores, Chiapas, se encontró que la especie predominante fue L. nasutus con 1,968 ejemplares (Pacheco-Flores, et al. 2008). Los adultos, de hábitos nocturnos, son atraídos a las luces eléctricas entre mayo y septiembre, pero se desconocen sus preferencias alimentarias y las de sus larvas (Morón et al., 1997). Por otra parte, King y Saunders (1984) observaron que en algunas localidades centroamericanas los adultos de L. nasutus a veces perforan las raíces del "tiquisque" (Xanthosoma spp. Araceae), cortan los tallos de la lechuga (Lactuca sativa L.) y otros cultivos durante junio y la primera parte de julio, y que las larvas se alimentan de las raíces del maíz y otros cultivos, debilitando y matando a la planta, pero el daño está restringido a agosto y septiembre. Las características morfológicas que distinguen a los estados inmaduros de L. nasutus son desconocidas, por lo que en el presente trabajo se describen por vez primera la larva de tercer estadio y la pupa y se aporta información sobre su biología.

\section{MATERIALES Y MÉTODOS}

Las larvas de tercer estadio utilizadas en la descripción de L. nasutus se colectaron durante 2006 en parcelas agrícolas donde había ganado alimentándose con rastrojo de maíz del ciclo anterior, y en potreros con ganado vacuno de Villa Flores, Chiapas. De la muestra de 47 larvas de tercer estadio obtenida en campo, diez se fijaron en solución de Pampel y se conservaron en alcohol etílico al 70 \%; y 37 larvas se criaron en cautiverio hasta obtener exuvias, pupas y adultos. Las larvas vivas se alimentaron cada tercer día con $18 \mathrm{~g}$ de estiércol seco de ganado vacuno, previamente humedecido con $5 \mathrm{ml}$ de agua. Las observaciones e ilustraciones se hicieron con ayuda de un estereomicroscopio Carl Zeiss Stemi SV6 usando los aumentos 0.8, 1.2, 2, 2.5, 4 y 5X; las mediciones se realizaron con un estereomicroscopio Nikon SMZU zoom 1:10, con los aumentos 1,2, 4,5 y 7.5X. Los caracteres y la terminología utilizada en las descripciones, son los propuestos por Boving (1942) y Morón (1986). Los ejemplares estudiados están depositados en la colección del proyecto "Diversificación en Sistemas de Cultivos" de ECOSUR-San Cristóbal de Las Casas, Chiapas.

\section{Ligyrus (Ligyrus) nasutus (Burmeister, 1847)}

(Figs. 1-15)

Larva de tercer estadio ( $\mathrm{N}=5)$. Longitud dorsal aproximada del cuerpo 42 a $54 \mathrm{~mm}$ en promedio, Cabeza parda rojiza, cuerpo blanco cremoso. Cabeza. Anchura máxima de la cápsula cefálica: 5.5-6 $\mathrm{mm}(\mathrm{N}=5)$. Superficie del cranium pardo rojiza con 
abundantes puntos amplios y profundos. Frente con 1-2 sedas en los ángulos anteriores, 1 seda-dorso-epicraneal, 0-1 seda epicraneal, y de 3-4 sedas paraocelares a cada lado. Carece de sedas frontales anteriores, posteriores y exteriores. Ocelos bien definidos (Fig.1). Clípeo con 2 sedas centrales y 1-2 sedas laterales a cada lado. Labro simétrico, rugoso, con 2-6 sedas posteriores, 2 sedas anteriores y 3 sedas laterales a cada lado. La epifaringe (Fig. 2) tiene una anchura de 2.25-2.4 mm y una longitud de 1.62-1.75. Carece de proplegmatia, plegmatia, clithra, dexiophoba y haptolachus. Haptomerum prominente con una placa esclerosada entera, sin heli. Pedium muy amplio. Acanthoparia con 9-12 sedas espiniformes. Corypha formada por 4-5 sedas. Acroparia con 5-6 sedas espiniformes. Dexiotorma larga y delgada. Laeotorma corta y estrecha. Placa esclerosada grande semitriangular. Cono sensorial ovalado, alargado. Chaetoparia izquierda con 55-88 sedas gruesas. Chaetoparia derecha con 47-62 sedas gruesas. Gymnoparia estrecha. Mandíbulas con un área estriduladora ventral, la mandíbula derecha (Fig. 3) con tres dentículos posteriores a la escotadura incisiva poco definidos, con cuatro lóbulos molares, acia reducida y bárbula escasa. Mandíbula izquierda (Fig. 4) con dos dentículos posteriores a la escotadura incisiva bien definidos, el proximal cuadrangular, calx alargado y bárbula abundante. Maxilas (Figs. 5-6) con un uncus en la galea y tres unci en la lacinia; área estriduladora maxilar formada por 8-10 dientecillos. Escleroma hipofaríngeo asimétrico muy esclerosado con el lado derecho prominente (Fig. 9). Último antenómero (Figs. 7-8) con 2 áreas sensoriales ovaladas dorsales y de 2-3 áreas sensoriales ventrales.

Tórax. Pronoto con un escleroma amarillo lateral alargado a cada lado, con 3-5 sedas. Proprescudo con 1-6 sedas cortas y largas, proescudo con 2 sedas largas transversales; mesoprescudo con 0-2 sedas largas transversales; mesoescudo sin sedas; mesoescutelo 0-2 sedas largas; metaprescudo con 1-4 sedas cortas y largas distribuidas irregularmente; metaescudo sin sedas; metaescutelo con 2 sedas largas transversales. Los estigmas respiratorios (Fig. 13) miden $0.44-0.53 \mathrm{~mm}$ de largo por 0.8-0.87 mm de ancho; placa respiratoria amarilla rojiza, uniformemente recurvada en forma de "C"; la distancia entre los lóbulos de la placa respiratoria es menor que el diámetro dorsoventral de la bulla; la bulla redondeada. Uñas tarsales alargadas, aguzadas, con una seda dorsal y una seda ventral prebasal (Figs.10-12). Uñas metatarsales más cortas que las precedentes (Fig. 12).

Abdomen. Estigmas abdominales de los segmentos I a VIII con diámetros similares, 0.4-0.65 de largo por 0.61-0.81 de ancho; placa respiratoria amarillo rojiza, regularmente recurvada en forma de "C". Dorsa de los segmentos abdominales I-VI con numerosas sedas espiniformes $y$ algunas sedas largas distribuidas transversalmente, el VII segmento con pocas sedas espiniformes y sedas largas transversalmente, los segmentos VIII-IX con sedas largas en hilera tranversal. Venter de los segmentos abdominales de I a IX con 1- 8 sedas medianas y largas en hileras 

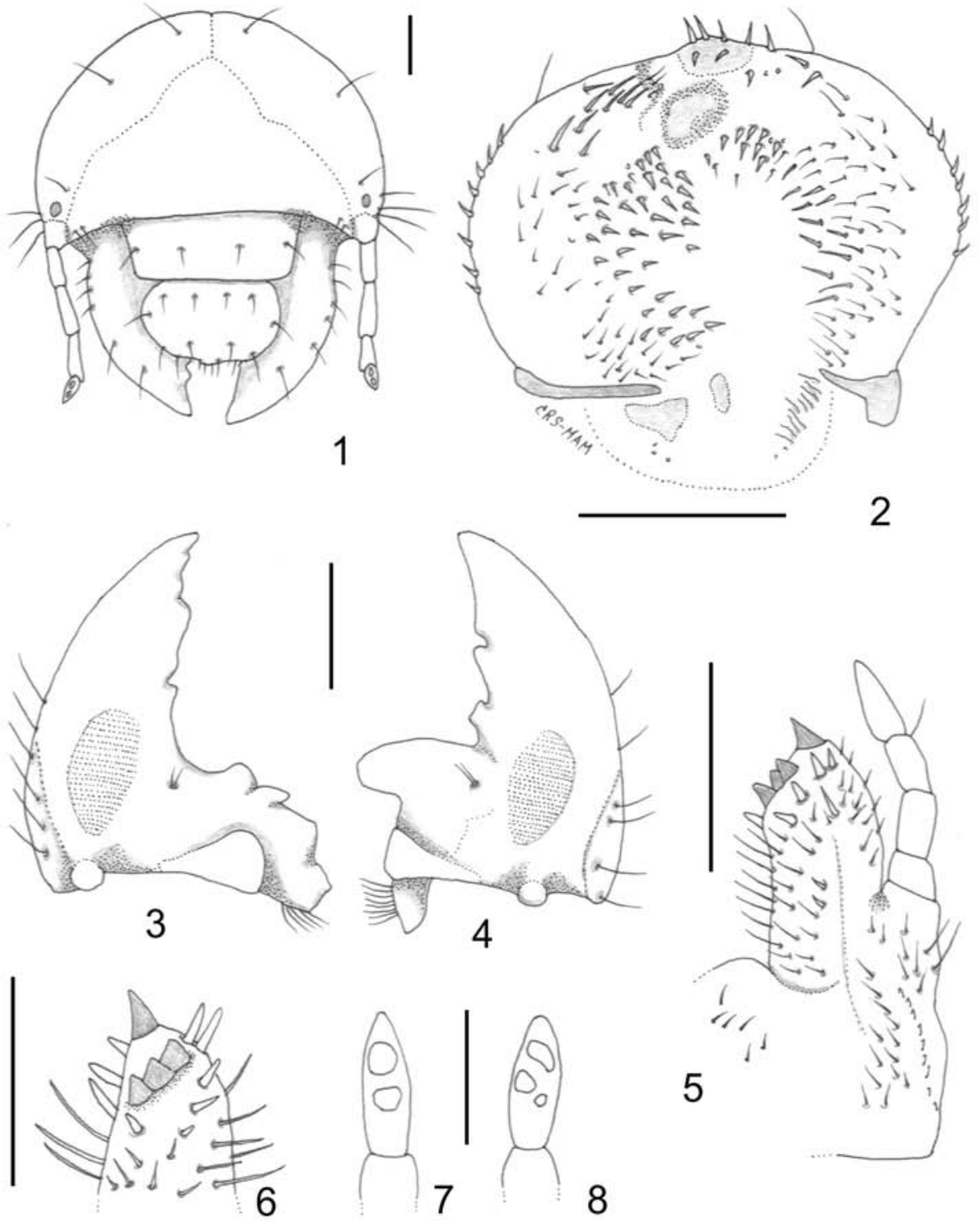

Figuras 1-8. Ligyrus nasutus. Larva de tercer estadio. 1) Vista frontal de la cabeza. 2) Epifaringe. Vista ventral de: 3) mandíbula derecha, 4) mandíbula izquierda. 5) Vista dorsal maxila izquierda. 6) Aspecto mesial del ápice de la maxila izquierda. Último antenómero: 7) dorsal. 8) ventral. Escalas: 1 $\mathrm{mm}$, excepto en figs. $7-8=0.5 \mathrm{~mm}$. 
transversales. Raster (Fig. 14) con una septula muy estrecha, pero larga, que se extiende sobre el labio anal inferior, en ocasiones no bien definida. Palidia formado por dos hileras irregulares de 13-16 pali, las cuales invaden el labio anal inferior. Labio anal inferior con 58-71 sedas espiniformes y algunas sedas largas. Abertura anal transversal moderadamente recurvada.

Pupa (N=2). Longitud corporal: 21-22 mm. Anchura humeral 9-10 mm. Cabeza. Glabra, fuertemente inclinada hacia abajo. Frente con depresiones irregulares, clípeo convexo, labro poco notable, piezas bucales claramente diferenciadas, ojos hundidos, tecae antenales engrosadas.

Tórax. Pronoto convexo. Meso y metanoto bien diferenciados. Tecae elitrales y tecae alares similares en tamaño. Protibias ligeramente más largos que los tarsos respectivos. Mesotibias tan largas como los mesotarsos. Metatibias un poco más largas que los tarsos respectivos.

Abdomen. Primeros seis segmentos claramente más anchos que los segmentos distales, con cinco pares de órganos dioneiformes bien definidos y esclerosados entre el dorso de los segmentos I-V (Fig.15). Estigma respiratorio del primer segmento no visible, cubierto por la base de las tecae alares. Estigmas de los segmentos II-IV con peritremas esclerosados muy prominentes. Estigmas de los segmentos V-VII cerrados, reducidos y rodeados de arrugas finas; el VIII estigma poco prominente, rodeado de arrugas finas. Último terguito abdominal sin urogomphi. Último esternito abdominal con una ámpula genital poco prominente en las hembras y muy notable en los machos.

Material examinado: cinco larvas de tercer estadio recolectadas en México. Estado de Chiapas, municipio de Villa Flores, 19-20-V-2006 y 14-VII-2006, C. Pacheco-Flores. Dos de ellas se recolectaron en estiércol y tres en parcela agrícola. De las larvas recolectadas en el mismo lugar el 22-V-06 se obtuvieron dos pupas en el laboratorio de bioensayos de ECOSUR-San Cristóbal.

Biología. Los adultos de L. nasutus se recolectaron con trampa de luz y bajo el alumbrado público. Las larvas se hallaron en las parcelas agrícolas donde se alimentó el ganado con el rastrojo del ciclo agrícola anterior y en los potreros. Con estiércol seco de ganado vacuno, humedecido ligeramente con agua, se alimentaron las larvas en el laboratorio. La cría de L. nasutus, a partir de las recolectas en campo, comprendió un periodo entre 51 y 56 días. Como larvas se mantuvieron 35 días (19 de mayo-25 de junio). El periodo pupal duró en promedio 16 días ( 22 de junio hasta 8 de julio). Los adultos comenzaron a emergen a partir del 6 de julio; estos son de color negro, miden $2.0 \mathrm{~cm}$ de largo y $0.9 \mathrm{~cm}$ de ancho. Cabeza: Ápice del clipeo acuminado, frente con una carina transversal, borde externo de la carina recurvado con dos dentículos apicales. Borde exterior de las protibias tridentado. Primer intervalo elitral uniformemente punteado (PachecoFlores, 2006). 


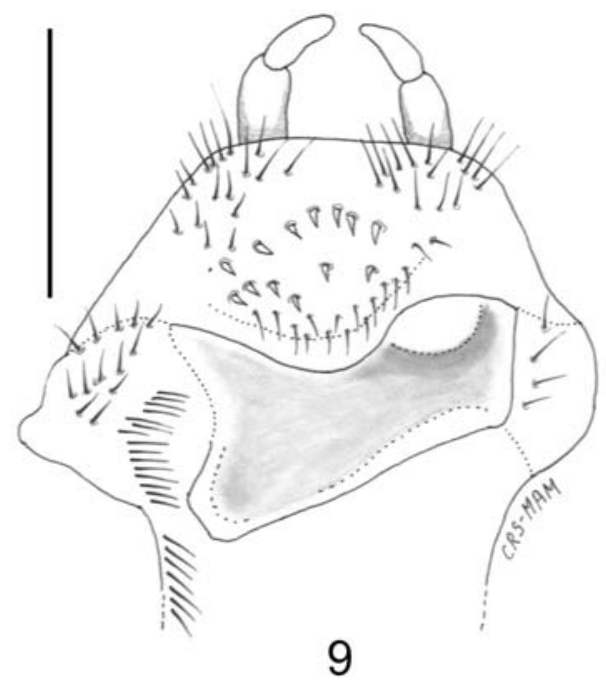

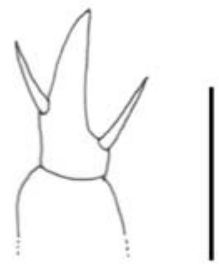

10

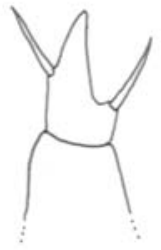

12

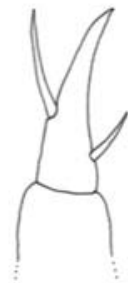

11

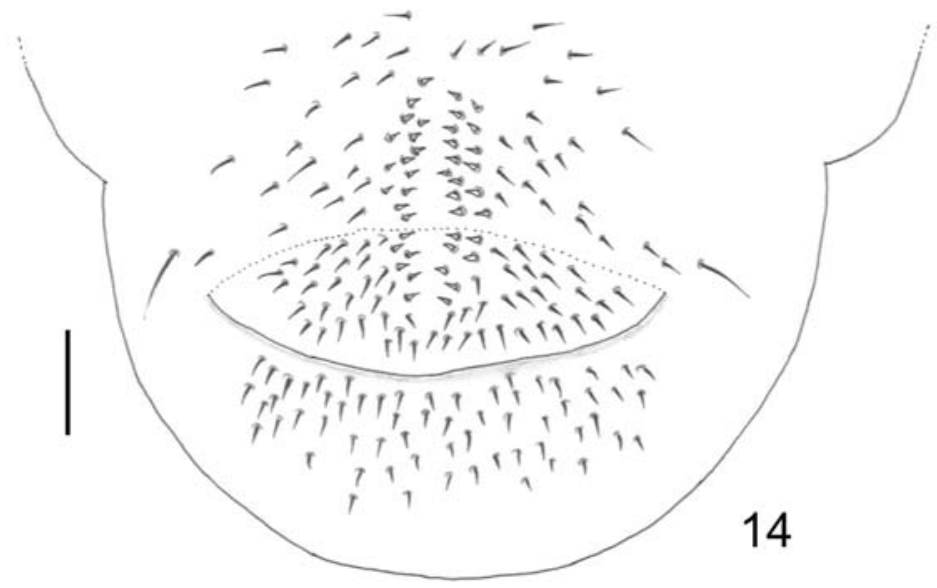

Figuras 9-14. Ligyrus nasutus. Larva de tercer estadio. 9) Hipofaringe. Uñas: 10) protarsal, 11) mesotarsal, 12) metatarsal. 13) Placa respiratoria protorácica. 14) Raster. 


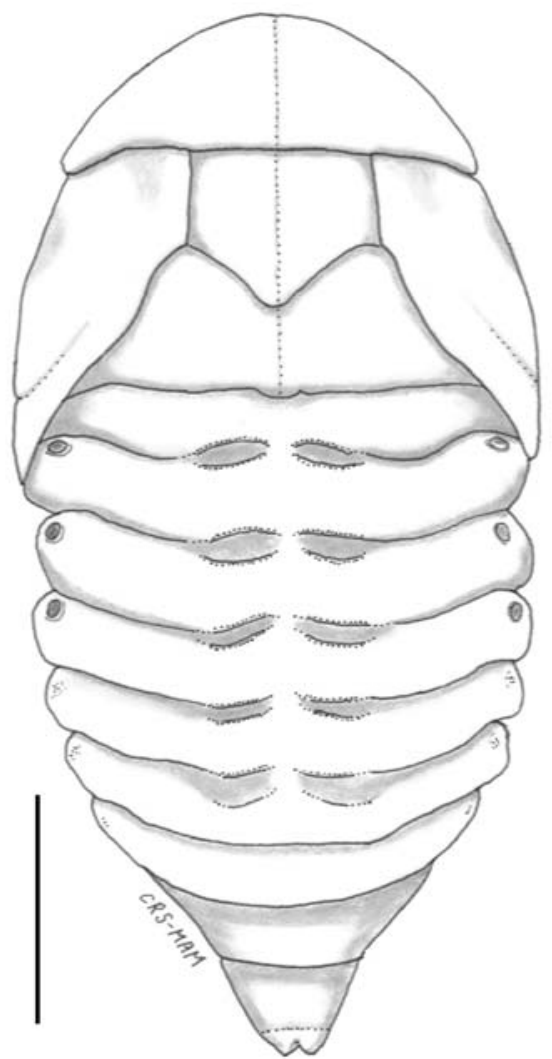

Figura 15. Ligyrus nasutus. Pupa. Aspecto dorsal. Escala: $5 \mathrm{~mm}$.

La abundancia de la larva está relacionada con la ganadería, ya que el estiércol de ganado, seco y humificado es parte importante de su alimentación. Además, las larvas de la especie tienen un papel importante en la degradación del estiércol seco, ya que durante las observaciones cuantitativas realizadas al alimentar a las larvas se observó que tienen la capacidad de procesar el estiércol de forma muy rápida, entre 252 y 270 g de estiércol por cada larva (Pacheco-Flores, 2006).

Comentarios taxonómicos. Considerando que en algunas localidades mexicanas es posible encontrar a Ligyrus nasutus y a $L$. sallei en condición parapátrica, a continuación señalamos algunas diferencias que permiten distinguir a las larvas de las dos especies con facilidad. La larva de L. nasutus es muy parecida a la de L. sallei, pero se diferencian en que la primera tiene,en el último artejo antenal 2 áreas sensoriales ovaladas dorsales y de 2-3 áreas sensoriales ventrales, haptomerum con 
placa esclerosada prominente entera, frente con 1-2 sedas en los ángulos anteriores, palidia con dos hileras irregulares de pali. La larva de $L$. sallei tiene en el último artejo antenal de 2-3 áreas sensoriales ovaladas dorsales y 3-4 áreas sensoriales ventrales, haptomerum prominente bidentado, frente con 2-3 sedas en los ángulos anteriores, palidia con 3-4 hileras irregulares de pali (Ramírez-Salinas et al., 2004).

\section{Clave para la identificación de las larvas de tercer estadio del género Ligyrus Burmeister basada en las descripciones de Ritcher (1966) y Ramírez-Salinas et al. (2004)}

1. Raster con palidia y septula que se extienden hasta el labio anal inferior. Cabeza sin sedas frontales posteriores ni anteriores................................................... 2 1' Raster sin palidia ni septula. Cabeza con una seda frontal posterior a cada lado. Estados Unidos y norte de México ....................... (Ligyrus) gibbosus (DeGeer)

2. Placa esclerosada del haptomerum bidentada o hendida. Cada palidium formado por 3-4 hileras irregulares de pali cortos. Texas, México y América Central.

$$
\text { L. (Ligyrodes) sallei Bates }
$$

2' Placa esclerosada del haptomerum entera. Cada palidium formado por 2 a 7 hileras

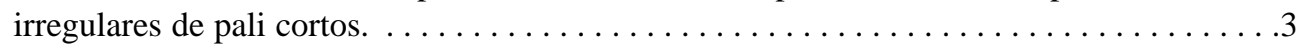

3. Cada palidium formado por dos hileras irregulares de pali cortos. Cabeza con 1.-2 sedas en cada ángulo antero-frontal. Occidente y sureste de México y América Central.

$$
\text { ................................... (Ligyrus) nasutus (Burmeister) }
$$

3' Cada palidium formado por 5-7 hileras irregulares de pali cortos. Cabeza com 2-3 sedas em cada ángulo antero-frontal. Sur de Estados Unidos y norte de México.

$$
\text { ..L. (Ligyrodes) relictus (Say) }
$$

AGRADECIMIENTOS. Al club de labranza de conservación "Innovadores de la Frailesca, A.C.” de Villaflores. Al CONACYT por la beca de estudios de posgrado otorgada al segundo autor. Este artículo derivó de la investigación financiada por el Programa de Apoyo a Tesis de Maestría de El Colegio de la Frontera Sur (PATM-ECOSUR) y la línea de investigación Diversidad en Sistemas de Cultivo de ECOSUR.

\section{LITERATURA CITADA}

Alcázar-Ruiz, J.A., Morón-Ríos, A. \& Morón, M.A. 2003. Fauna de Coleoptera Melolonthidae de Villa Las Rosas, Chiapas, México. Acta Zoológica Mexicana (n. s.), 88: 59-86.

Boving, A. 1942. A classification of larvae and adults of the genus Phyllophaga (Coleoptera: Scarabaeidae). Memoires of the Entomological Society ofWashington, 2: 1-95.

King, A.B.S. \& J. L. Saunders. 1984. Las plagas invertebradas de cultivos anuales alimenticios en América Central. TDRI-ODA, CATIE, Londres. $182 \mathrm{pp}$.

Morón, M. A. 1986. El género Phyllophaga en México. Morfología, distribución y sistemática supraespecífica (Insecta: Coleoptera). Pub.19. Instituto de Ecología. México. 344 pp. 
Morón, M.A., Ratcliffe, B.C. \& Deloya, C. 1997. Atlas de los escarabajos de México, Coleoptera: Lamellicornia. Vol. I Familia Melolonthidae. Publ. Esp. de la Sociedad Mexicana de Entomología A. C. y CONABIO. México. 264 pp.

Morales-Morales, C.J., Rosales-Esquinca, M. de los A., Ponce-Díaz, P., Gutiérrez-Hernández, R.C., Quiroga-Madrigal, R.R. \& Zuart-Macias, J.L. 2005. Melolóntidos (Coleoptera: Scarabaeoidea) de la zona de conservación ecológica Laguna Bélgica, Ocozocuautla, Chiapas, México. Entomología Mexicana, 4: 144-148.

Pacheco-Flores, C. 2006. Diversidad de escarabajos melolóntidos (Coleoptera: Scarabaeoidea) en el municipio de Villaflores, Chiapas, México. Tesis de Maestría en Ciencias. El Colegio de la Frontera Sur, Chiapas, México. 113 pp.

Pacheco-Flores, C., A. E. Castro-Ramírez, M. A. Morón y B. Gómez y Gómez. 2008. Fauna de escarabajos melolóntidos (Coleoptera: Scarabaeoidea) en el municipio de Villaflores, Chiapas, México. Acta Zoológica Mexicana (n. s.), 24(1): 139-168.

Ramírez-Salinas, C., M. A. Morón \& A. E. Castro-Ramírez. 2004. Descripción de los estados inmaduros de tres especies de Anomala, Ancognatha y Ligyrus (Coleoptera: Melolonthidae: Rutelinae y Dynastinae) con observaciones de su biología. Acta Zoológica Mexicana (n.s.), 20(3): 67-82.

Ritcher, P. O. 1966. White grubs and their allies: a study of the North American scarabaeoid larvae. Studies in Entomology No. 4. Oregon State University Press. Corvallis. 